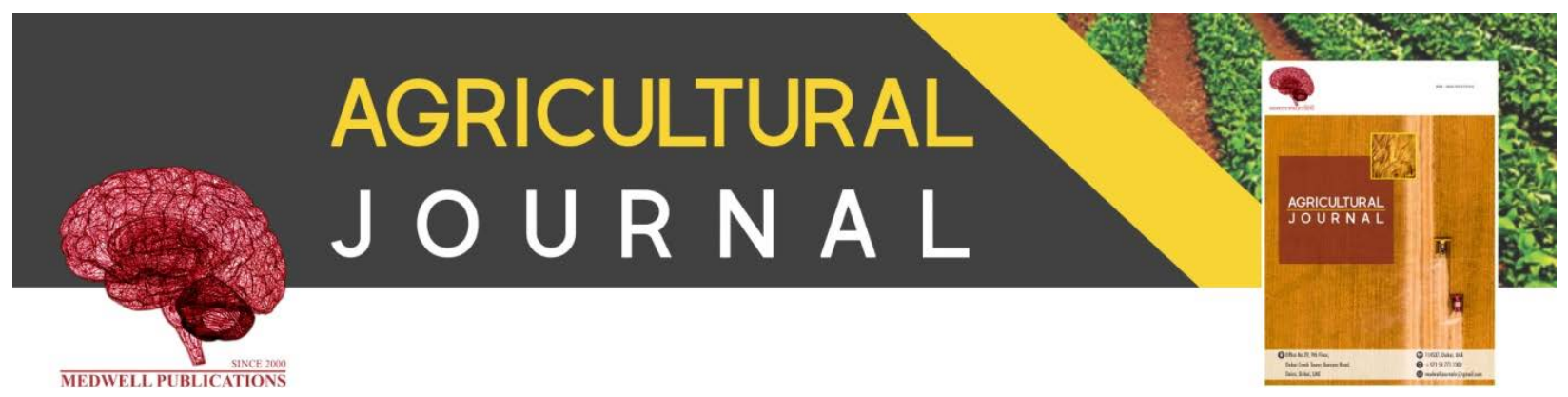

\title{
Rice Blast (Magnaporthe oryzae) Management: A Review
}

${ }^{1}$ Koshraj Upadhyay and ${ }^{2}$ Bhupendra Bhatta

${ }^{1}$ Institute of Agriculture and Animal Science, Kirtipur, Kathmandu, Nepal

${ }^{2}$ Univesity of Idaho, Moscow, 83843 Idaho, USA

Key words: Control, disease, epidemiology, Magnaporthe oryzae, rice, symptoms

\section{Corresponding Author:}

Koshraj Upadhyay

Institute of Agriculture and Animal Science, Kirtipur, Kathmandu, Nepal

Page No.: 42-48

Volume: 15, Issue 4, 2020

ISSN: 1816-9155

Agricultural Journal

Copy Right: Medwell Publications
Abstract: Rice blast caused by M. oryzae is one of the most devastating fungal disease of rice in the world. The crop suffers from the chronic fungal pathogen from seedling to adult plant stages affecting leaves, collar, node, panicle, neck, roots, seeds, etc. The main objectives of this review paper is to know the symptoms of rice blast disease, its epidemics and different measures of effective disease control. Resistant varieties and chemical control are most effective means of disease control. Findings shows $>100$ resistant genes and 350 quantative trait loci's for blast resistant has been identified in rice. Extensively used persistant chemicals should replaced by Neem based commercial pesticides and biocontrol agents like Pseudomonas fluorescens, Streptomyces for sustainable and ecofriendly control of rice blast havoc. Disease forecasting methods developed by researchers provides pre-information of disease occurrence that helps to design control measures combination for effective management of the disease.

\section{INTRODUCTION}

Rice (Oryza sativa L.) is the major food crop for the world's population and Asia accounts for about $90 \%$ of the world's rice production and consumption. Globally, rice is cultivated in 160 million hectares with production of 741 million tons (Food and Agriculture Organization (FAO, 2016)). Among several diseases of rice caused by fungal, bacterial and viral pathogens, rice blast is one of the most significant diseases economically. Rice blast is observed in almost all rice growing countries causing annual yield loss up to $30 \%$ which is equivalent to feeding 60 million people (Skamnioti and Gurr, 2009). Yield loss and mitigation cost account for high economic loss due to blast disease. In the US alone, yield loss due to blast disease is estimated to be sufficient to feed a million people per year (Nalley et al., 2016). As the US is a small producer of rice and has less loss from blast than the global average, it can be inferred that total yield loss due to blast disease in the world is of a significant amount.

The ascomycete fungus, Magnaporthe oryzae (anamorph Pyricularia oryzae) causes the rice blast disease. M. oryzae is a hemibiotroph that establishes a biotrophic relationship with the host initially and necrotrophic association later. It weakens the plant defense system without producing visible symptoms during biotrophic association and promotes cell death when it shifts to the necrotrophic association (Fernandez and Orth, 2018). The pathogenis able to infect and produce symptoms in all parts of the plant.The disease appears in the form of leaf blast, panicle blast or neck rot, collar rot and node blast (Iwata, 2001). Although, lesions can be observed on all infected plant 
Agric. J., 15 (4): 42-48, 2020

parts including seeds and roots, diamond-shaped lesions produced on the leaves are the diagnostic symptom. Infection to the panicle can reduce seed set causing yield loss of $0.5 \%$ for every $1 \%$ of rotten neck and can lead to seed infection as well (TeBeest et al., 2012). It is also found that there is a $22 \%$ increase in odds for seed infection with a $10 \%$ increase in neck blast (Manandhar et al., 1998a). Due to these reasons, panicle blast or neck rots are considered the most destructive part of the disease.

\section{IDENTIFICATION OF RICE BLAST}

Rice blast can be identified on the basis of presence of lesions on plant parts that includes leaf, leaf collars, necks, panicles, pedicles, seeds, etc. Recent findings show that roots of rice plant also have lesions. The most common and distinct symptoms of rice blast occurrence is 'diamond shaped lesions' on leaf surface of rice plant.

Symptoms on leaves: Rice leaves are most and readily affected part of rice plant. Symptoms of rice blast in the leaves varies with the agroclimatic and environmental state, age of the plant and resistance level of the host cultivar. On susceptible cultivar, gray green and water-soaked lesions appear initially with darker green border which expands rapidly to several centimeters long. Older lesions on susceptible varieties becomes light tan in color with necrotic boarders. Resistant varieties are characterized by presence of small size (1-2 $\mathrm{mm})$ lesions with brown to dark brown color.

Symptoms in rice collar: Area of necrosis at the union of leaf blade and stem sheath is symptoms of rice collar infection. This infection can kill the entire leaf and expands to few millimeters on stem sheath.

Symptoms in rice neck and panicles: The portion of stem that rises above the upper leaf which supports panicle is rice neck. Magnaporthe oryzae infects rice neck at the node that leads to a condition called rotten neck or neck blast. Infection on neck are disastrous that develops chaffy grains due to failure of seed to fill in the case of neck rot entire panicle falls down. In case of panicle infection gray brown lesions can be easily found on panicle branches, spikes and spikelet. Over time panicle branches breaks at the lesion presence spot.

Symptoms on rice seeds: Seed surface of infected rice panicle has brown spots, blotches and sometimes diamond shaped lesions as seen in leaves. The fungus is found to be present in pedicles of the seed resulting blank seed of rice and the condition is known as blanking. Recent findings believe that blast fungus can infect seeds through florets as they develop into seeds. The full process and time of seeds infection by spores of pathogen is still unknown.

\section{DISEASE CYCLE AND EPIDEMIOLOGY}

M. oryzae overwinters on crop debris, seeds and on weed hosts. The pathogen overwinters in the form of mycelium in crop debris and as conidia on the living hosts. Although, infected crop debris is a major source of primary inoculum, infested seeds are also considered the important source (Thurtson, 1995). Infested seeds are produced when the plant is inoculated at any stages after the flag leaf is fully developed. The infested seeds produce diseased seedlings which die and serve as primary inoculum (Faivre-Rampant et al., 2013). Conidia are produced and released by overwintering fungus during the period of high relative humidity ( $>90 \%)$. The airborne conidia land on rice plant and adhere strongly through the mucilage they produce at their tip. In wet conditions, conidia germinate to form a germ tube which later produces specialized structures called appressorium. Deposition of melanin and recycled conidium contents inside the appressorium creates internal turgor pressure of up to 8.0 MPa which is enough to penetrate the rice cells by the formation of penetration peg (Fernandez and Orth, 2018). At optimum temperatures, lesions appear within 4 to 5 days. In wet weather, new conidia are produced within an hour from the appearance of lesions and most of them are released between midnight and sunrise. These new conidia germinate and cause secondary infection (Agrios, 1997). The secondary cycles can be repeated many times during the growing season depending upon the environmental and growing conditions. Prolonged wetness, high humidity, high nitrogen application and moderate temperature $\left(24^{\circ} \mathrm{C}\right)$ favors the disease development (TeBeest et al., 2012).

\section{STRATEGIES TO CONTROL}

Distribution of rice blast disease in almost all rice growing areas and wide host range of $M$. oryzae makes eradication ofthe disease difficult. In fact, rice blast has never been eliminated from a region in which rice is grown (TeBeest et al., 2012). Cultural methods were the only tool to control the blast disease in the past when chemicals and resistant varieties were not available. These methods are effective in creating a less favorable environment for the pathogen in the field and reducing the blast disease epidemics. In areas of low blast pressure, control of rice blast is primarily based on the use of resistant varieties (Agrios, 1997). Chemicals with specific fungicidal effects on the blast pathogen are used to control the disease. Biological control agents are used as an eco-friendly strategy for rice blast management. Rice blast control strategies and techniques that have been most effectively utilized will be explored in this study. These strategies can be broadly classified as cultural control, resistance, chemical control and biological control. 
Forecasting blast disease: Disease forecasting is one of the important tools to select prevention and control measures. Epidemiological study of disease helps to select the efficient disease management tool. Van der Plank emphasizes epidemiological study is crucial for selection of effective disease management tools developed by plant breeders and chemical industries.

For most devastating disease of rice, some blast forecasting models has been developed to predict its occurrence and severity to control the disease in an effective way. Due to uncertainties and inaccuracy of in prediction disease forecasting methods are not widely used.

Disease forecasting focus on prediction of probable outbreak of the pathogen or increase intensity of disease that allows when how and where a specific disease management practice should be done (Agrios, 1997). Forecasting methods relies on the host, pathogen and environment condition for disease development, host must be susceptible, pathogen virulent and environment favorable.

Researchers have suggested most epidemic models are either analytical or simulation. Analytical measures are simple and considers only few variables whereas simulation measures consist of series of equations. Berger observed that some researchers combined two approaches starting with analytical models and gradually increasing degree of confidence with breaking down to the complex models in analytical forms.

Use of botanicals: Chemicals are extensively used for management of blast disease in rice. However, those chemicals use resulted in environmental pollution and affect health of biotic community. So, there is urge to develop natural products alternatives for those non-biodegradable, persistent chemicals. The botanicals are biodegradable, target specific, environmentally friendly, relatively cheap and easy to use.

Nowadays, ecofriendly chemicals methods like organomercuric, organophosphorus, copper fungicides, antibiotics like Kasugamycin/Blasticidin and leaf extracts are used to control blast disease effectively. Neem based pesticides spray, plant dried commercial products like Achoole, Neemzal, Neem Gold and Neem Azal T/S are promising botanicals in reducing severity of rice blast (Pandey, 2018). Neem seed kernel extract is considered as 2nd best method of leaf blast control after pseudomonas and fluoresce. Leaf extract of Calotropis, calendula, Eclipta alba, moringa, etc., like medicinal plants are said to have lowered the severity of blast disease (Jyotsna et al., 2017). Combination of bael and tulsi leaf extracts with chemicals is also one of the effective methods of controlling leaf blast. Work done by Amadioha (2000) found that commercial botanicals products with principle ingredient as azadiractin is effective in blast management (Amadioha, 2000). Neem based commercial botanicals plays significant role in preventing germination of blast fungal spores. Recent study confirms the antifungal activity of some botanicals against $M$. oryzae.

Cultural practices: Excessive application of nitrogen fertilizer, water stress, the presence of infected debris in the field and use of infested seeds are the factors that favor the occurrence and spread of the disease. Destroying diseased crop debris reduce the overwintering inoculum in the field. Application of nitrogen above the recommended rate and as a single application significantly increases disease incidence and disease severity. Average leaf blast incidences at the panicle primordia stage were $73 \%$ in high-N, $60 \%$ in normal-N and $43 \%$ in split-N treatments (Long et al., 2000). Therefore, it is recommended to apply an optimum dose of nitrogen in split doses based on soil testing results. It is known that Silicon (Si) reduces the frequency of appressorial penetration by the blast fungus. Results of work by Hayasaka et al. (2008) have suggested that the proportion of appressorial penetration after $111 \mathrm{~h}$ decreased 5-fold and the proportion of penetration that developed to lesions decreased 2.5 fold when the amount of Si application increased from 0-0.8 g per plant.

Therefore, it is suggested that the application of $\mathrm{Si}$ is found to have a positive effect on the resistance of rice plant against blast disease. As high humidity favors blast disease, planting time should be selected to avoid flowering coinciding with periods of high humidity. Use of healthy seeds reduces seed borne infection. Flooding of the field to avoid water stress can reduce disease development. The anaerobic condition is unfavorable for the blast pathogen. It is observed that the complete covering of seeds or seeding underwater results in reduced seed-borne infection by blastpathogen and transmission to seedlings (Manandhar et al., 1998a, b).

Resistant varieties: Although, different measures are used to control the rice blast disease, planting blast-resistant variety is considered as the most effective method by farmers (Leung et al., 2003). To date, about 100 quantitative blast Resistance (R) genes and more than 350 quantitative trait loci QTLs have been identified in rice and 19R genes have been successfully cloned and characterized (Ballini et al., 2008; Sharma et al., 2012). Blast resistant rice varieties have been developed by using conventional breeding approaches that include pedigree method, backcrossing, recurrent selection and mutation breeding. More recent approaches being used are marker-assisted selection, allele mining, association mapping, genome editing, genetic transformation and nanotechnology (Srivastava et al., 2017). A single 
dominant $R$ gene is effective in preventing disease by $M$. oryzae containing corresponding Avirulence (AVR) gene (Silue et al., 1992). However, the breakdown of resistance to the disease has occurred by new races of blast fungus and the resistant varieties must be changed frequently. The stability and efficacy of resistance due to the $R$ gene (Pi-ta) is determined by the changes in the functional region of the corresponding $A V R$ gene (AVR-Pita) of $M$. oryzae. It is found that transposon insertion into the coding region of AVR-Pita gene might be the reason for the loss of rice blast resistance due to $R$ (Pi-ta) gene in rice cultivars (Zhou et al., 2007). Pyramiding the $\mathrm{R}$ genes in rice cultivars is one of the strategies to overcome the relatively small effects of single $R$ genes and further improve the resistance to rice blast. Research by Yasuda etc. has suggested that the combination of two resistance genes is either more effective than the genes individually or similar to the level of the most effective resistance gene in the pair based on the combination of genes and characteristics of infection. To maintain high yield is another challenge while breeding for disease resistance. Recently, it has been found that balancing high disease resistance and yield can be done through epigenetic regulation of paired antagonistic Nucleotide-binding Leucine-Rich (NLR) receptors (Deng et al., 2017).

Selection of resistant variety varies from one location to another and from one season to other in the same location as well. A number of resistant varieties are identified and used for breeding programs. Due to variation in pathogenic races at different times and places, no varieties seem to be resistant to all races. Also, it should be kept in mind that no pathogen race has the ability to infect all the varieties of rice (Ou, 1985).

Chemical control: Use of chemicals, mainly fungicides and antibiotics, to control the rice blast disease is based on two techniques: seed treatment prior to sowing and foliar spraying or dusting of rice plants in the field. The development of chemical control for rice blast can be traced by studying the chemical control program of Japan. Copper fungicides were used to control the blast disease until after the Second World War. However, high phytotoxicity of copper-based fungicides resulted in yield reduction and the organomercuric compounds rapidly replaced copper-based fungicides during the 1950's. Although, organomercuric compounds had very low phytotoxicity and were highly effective in controlling blast, they were banned by the Japanese government during the mid-1960's due to heavy metal poisoning. Systemic antibiotic (Blastidin-S) was then intensively used to control blast disease. The development and use of organophosphorus fungicides took place at about the same time as antibiotics. According to a study by Uesugi blast pathogen showed resistance to the antibiotic compounds and organophosphorus fungicides due to intensive use. This study suggested that the resistant population dropped to zero when the use of antibiotics is halted. A wide range of systemic fungicides with different mode of actions is used for rice blast control. Majority of the fungicides which are effectively used to control the rice blast disease are grouped as Plant Defense Activators (PDAs), Melanin Biosynthetic Inhibitors (MBIs), Chlorine Biosynthesis Fungicides (CBIs) and quinol site mitochondrial respiration inhibitors (Klittich, 2008; Yamaguchi, 2004).

The efficacy of these group of fungicides highly depends upon the type of fungicide used time and method of application, disease level during application, the efficiency of forecasting systems and by the presence and/or the emergence of fungicide-resistant strains. Using fungicides with different mode of action in the rotation or in combination ensures the better efficacy of fungicide and also lowers the emergence of fungicide-resistant strains.

Biological control: An effective biological control approach is self-sustaining, efficient and usually more enduring. Reduction of disease by biological control method is possible by a reduction in inoculum of the pathogen (decreased production and release of viable spores, decreased survival and decreased spread), reduction of infection of the host by the pathogen and reduction of severity of attack by a pathogen. Biocontrol of rice blast mainly relies on the use of antagonistic bacteria and induction of host resistance. Strain Pf 7-14 of Pseudomonas fluorescens was found promising in reducing blast disease significantly in field experiment by Gnanamanickam and Mew (1992). The result of this experiment showed that strains designated Pf4-15 and Pf 7-14 of P. fluorescens accounted for 59 and 47\% leaf blast reduction, respectively. Similarly, the Bacillus strains, $4-03$ and 33 gave 46 and $44 \%$ leaf blast reduction, respectively. The suppression of disease by $P$. fluorescens was likely due to antifungal antibiotics (AFA) which afforded $70-100 \%$ inhibition in conidial germination of blast fungus at $1.0 \mathrm{ppm}$ concentration. Seed treatment with Trichoderma harzianum (at $4 \mathrm{~g} \mathrm{~kg}^{-1}$ seed) is found to reduce the intensity of rice blast disease by $10-25 \%$ (Singh et al., 2012). Results have suggested that streptomyces inhibits the rice blast disease. Treatment of infected rice seedlings with streptomyces showed 88.3\% reduction of rice blast disease (Law et al., 2017). There are several naturally occurring rice rhizobacteria which are found to suppress rice blast infections. Spence et al. (2014) isolated and identified such bacteria and found that a pseudomonas isolate, EA105, significantly reduced the disease through reducing appressoria formation (by 90\%) and by inhibiting fungal growth by $76 \%$.

Several plant growths promoting rhizobacteria are found to induce systemic resistance against rice blast. Induced Systemic Resistance (ISR) is one of the mechanisms of blast disease suppression by $P$. 
Agric. J., 15 (4): 42-48, 2020

fluorescens 7-14 and P. putida V14i. It is found that the increased salicylic acid levels due to bacteria-induced ISR contributes in the suppression of rice blast by $25 \%$ (Krishnamurthy and Gnanamanickam, 1998). Blast resistance in rice is also induced by using avirulent strains of Pyricularia spp. against highly virulent strains of the pathogen. A study by Arase and Fujita (1992) showed that pre-inoculation with non-pathogenic Pyricularia spp. induced inaccessibility of pathogen in rice leaf-sheath cells thereby making the plant highly resistant to blast disease.

Foliar sprays of an avirulent strain of Pyricularia oryzae and isolates of Bipolaris sorokinianaat four leaf stages is found to induce systemic resistance and reduce rice blast disease by 20.3 and $30.2 \%$, respectively (Manandhar et al., 1998b).

The success and effectiveness of biological control depend on the ability of the biocontrol agent to survive and establish itself in the introduced environment. One of the challenges in the use of biocontrol agents is to make the formulation of biocontrol agents in such a way that these agents are preserved in a viable stage and are easy to handle during transport, storage and application (Gnanamanickam, 2002).

\section{CONCLUSION}

Resistant varieties and chemical control are the most effective means to control rice blast to the date. Although, complete eradication of the disease is not provided by cultural methods, these tools should always be considered for the management of the blast disease. Use of appropriate cultural practices is found promising in reducing primary inoculum and introduction of the pathogen in the new area thereby reducing the disease incidence. Biological control agents, mainly PGPRs are found successful against rice blast pathogen. As mentioned above, there are several disease control strategies and techniques which have been developed to control rice blast disease but they offer limited success (TeBeest et al., 2012). Selection of appropriate strategy and integrated use of best management practices is therefore, crucial for successful management of rice blast.

As M. oryzae is variable in nature, there is a need for continuous research to control blast disease. Further, research needs to be directed to develop high yielding, broad-spectrum resistant varieties and more effective chemical control. The research by Yasuda etc. and Dang et al. (2017) have opened possibilities in breeding highly resistant varieties with high yield. Further, work on searching for $\mathrm{R}$ genes that recognize $A V R$ genes and pyramiding those $\mathrm{R}$ genes can be promising to improve the sustainability of resistance to rice blast. Research on chemical control should be directed towards the development of broad-spectrum fungicides which are effective at lower doses and have a lower impact on the environment. Researches show that biocontrol agents are effective for controlling rice blast disease. However, formulation and cost associated with the production make use of biocontrol agents challenging in field conditions. Further research is needed to make formulations to facilitate transport and storage of biocontrol agents. The research focused on understanding the recognition, signaling and interaction between the biocontrol agents (mainly PGPRs) and pathogen will lead to the development of more precise and consistent strategies in the future. Research by Spence et al. (2014) shows that naturally occulting rhizobacteria (strain EA105 of P. chlororaphis in particular) can be useful to significantly reduce rice blast disease. More studies should be directed to isolate and identify the potential naturally occurring rhizobacteria against the rice blast disease and utilize them in the field. Most of the strategies of biocontrol involve the use of a single microorganism. Use of a combination of microorganisms as biocontrol agent may be more effective than a single culture due to a combined effect on rice blast pathogen and is a potential area of future research. The effectiveness of the blast disease control methods is based on information by disease forecasting systems. Accurate information by a reliable forecasting system will allow to the timely application of disease control strategies. However, the majority of the rice blast forecasting systems in practice are capable of predicting leaf blast only and usage of the models is limited due to inaccuracy in the information (Katsantonis et al., 2017). Thus, research directed towards the development of a reliable and accurate blast forecasting system has much potential for study and will be crucial to control rice blast disease in the future.

\section{REFERENCES}

Agrios, G.N., 1997. Plant Pathology. 4th Edn., Academic Press, San Diego, CA., USA., ISBN-13: 9780120445646, Pages: 635.

Amadioha, A.C., 2000. Controlling rice blast in vitro and in vivo with extracts of Azadirachta indica. Crop Protect., 19: 287-290.

Arase, S. and K. Fujita, 1992. Induction of Inaccessibility to Pyricuhria oryzae by Pre-inoculation of $\mathrm{P}$. grisea, in rice leaf-sheath cells. J. Phytopathol., 134: 97-102. 
Agric. J., 15 (4): 42-48, 2020

Baker, K.F. and R.J. Cook, 1974. Biological Control of Plant Pathogens. In: The Biology of Plant Pathogens, Kelman, A and L. Sequira (Eds.). W.H. Freeman and Co, San Francisco, California, pp: 220-285.

Ballini, E., J.B. Morel, G. Droc, A. Price, B. Courtois, J.L. Notteghem and D. Tharreau, 2008. A genome-wide meta-analysis of rice blast resistance genes and quantitative trait loci provides new insights into partial and complete resistance. Mol. Plant-Microbe Interact., 21: 859-868.

Deng, Y., K. Zhai, Z. Xie, D. Yang and X. Zhu et al., 2017. Epigenetic regulation of antagonistic receptors confers rice blast resistance with yield balance. Sci., 355: 962-965.

Faivre-Rampant, O., L. Genies, P. Piffanelli and D. Tharreau, 2013. Transmission of rice blast from seeds to adult plants in a non-systemic way. Plant Pathol., 62: 879-887.

Fernandez, J. and K. Orth, 2018. Rise of a cereal killer: The biology of Magnaporthe oryzae biotrophic growth. Trends Microbial., 26: 582-597.

Gnanamanickam, S.S., 2002. Biological Control of Crop Diseases. Marcel Dekker Inc., New York, USA., ISBN: 9780203910955, Pages: 480.

Gnananmanickam, S.S. and T.W. Mew, 1992. Biological control of blast disease of rice (Oryza sativa L.) with antagonistic bacteria and its mediation by Pseudomonas antibiotic. Ann. Phytopathol. Soc. Japan, 58: 380-385.

Hayasaka, T., H. Fujii and K. Ishiguro, 2008. The role of silicon in preventing appressorial penetration by the rice blast fungus. Phytopathology, 98: 1038-1044.

Iwata, M., 2001. Rice blast control. Royal Soc. Chem., 1: 3-5.

Jyotsna, J., S. Das and B. Kumar, 2017. Efficacy of aqueous leaf extract of medicinal plants against blast and brown spot disease of rice. Int. J. Curr. Microbiol. Applied Sci., 6: 4138-4144.

Katsantonis, D., K. Kadoglidou, C. Dramalis and P. Puigdollers, 2017. Rice blast forecasting models and their practical value: A review. Phytopathologia Mediterr., 56: 187-216.

Klittich, C.J., 2008. Milestones in fungicide discovery: Chemistry that changed agriculture. Plant Health Progress, Vol. 9,

Krishnamurthy, K. and S.S. Gnanamanickam, 1998. Induction of systemic resistance and salicylic acid accumulation in Oryza sativa L. in the biological suppression of rice blast caused by treatments with Pseudomonas spp. World J. Microbiol. Biotechnol., 14: 935-937.
Law, J.W.F., H.L. Ser, T.M. Khan, L.H. Chuah and P. Pusparajah et al., 2017. The potential of Streptomyces as biocontrol agents against the rice blast fungus, Magnaporthe oryzae (Pyricularia oryzae). Front. Microbiol., Vol. 8. 10.3389/fmicb.2017.00003

Leung, H., Y. Zhu, I. Revilla-Molina, J.X. Fan and H. Chen et al., 2003. Using genetic diversity to achieve sustainable rice disease management. Plant Disease, 87: 1156-1169.

Long, D.H., F.N. Lee and D.O. TeBeest, 2000. Effect of nitrogen fertilization on disease progress of rice blast on susceptible and resistant cultivars. Plant Dis., 84: 403-409.

Manandhar, H.K., H.J.L. Jorgensen, S.B. Mathur and V. Smedegaard-Petersen, 1998. Suppression of rice blast by preinoculation with avirulent Pyricularia oryzae and the nonrice pathogen Bipolaris sorokiniana. Phytopathol., 88: 735-739.

Manandhar, H.K., H.J.L. Jorgensen, V. Smedegaard-Petersen and S.B. Mathur, 1998. Seedborne infection of rice by Pyricularia oryzae and its transmission to seedlings. Plant Dis., 82: 1093-1099.

Nalley, L., F. Tsiboe, A. Durand-Morat, A. Shew and G. Thoma, 2016. Economic and environmental impact of rice blast pathogen (Magnaporthe oryzae) alleviation in the United States. PloS One, Vol. 11, No. 12. 10.1371/journal.pone.0167295

Ou, S.H., 1985. Rice Diseases. 2nd Edn., Commonwealth Mycological Institute, Kew, UK., Pages: 380.

Pandey, S., 2018. Effect of botanicals for management of leaf blast and enhancing yield traits in rice. Curr. Bot., 9: 17-21.

Sharma, T.R., A.K. Rai, S.K. Gupta, J. Vijayan, B.N. Devanna and S. Ray, 2012. Rice blast management through host-plant resistance: Retrospect and prospects. Agric. Res., 1: 37-52.

Silue, D., J.L. Notteghem and D. Tharreau, 1992. Evidence of a gene-for-gene relationship in the Oryza sativa-Magnaporthe grisea pathosystem. Phytopathol., 82: 577-580.

Singh, P.K., A.K. Singh, H.B. Singh and B.K. Dhakad, 2012. Biological control of rice blast disease with Trichoderma harzianum in direct seeded rice under medium low land rainfed conditions. Environ. Ecol., 30: 834-837.

Skamnioti, P. and S.J. Gurr, 2009. Against the grain: Safeguarding rice from rice blast disease. Trends Biotechnol., 27: 141-150.

Spence, C., E. Alff, C. Johnson, C. Ramos, N. Donofrio, V. Sundaresan and H. Bais, 2014. Natural rice rhizospheric microbes suppress rice blast infections. BMC Plant Biol., Vol. 14, 
Srivastava, D., M. Shamim, M. Kumar, A. Mishra and P. Pandey et al., 2017. Current status of conventional and molecular interventions for blast resistance in rice. Rice Sci., 24: 299-321.

TeBeest, D.O., C. Guerber and M. Ditmore, 2012. Rice blast. Plant Health Instructor, Vol. 1, 10.1094/PHI-I-2007-0313-07

Thurston, H.D., 1995. Tropical Plant Diseases. APS Press, Minnesota.
Yamaguchi, I., 2004. Overview on the Chemical Control of Rice Blast Disease. In: Rice Blast: Interaction with Rice and Control, Kawasaki, S. (Ed.)., Springer, Dordrechet, The Netherlands, pp: 1-13.

Zhou, E., Y. Jia, P. Singh, J.C. Correll and F.N. Lee, 2007. Instability of the Magnaporthe oryzae avirulence gene AVR-Pita alters virulence. Fungal Genet. Biol., 44: 1024-1034. 